\title{
重物认知信息对搬举任务生物力学特性的影响
}

\author{
刘一鹏, 刘骏识, 曲行达 \\ 深圳大学人因工程研究所，广东深圳 518060
}

\begin{abstract}
摘 要: 手工搬举导致的下背疼痛是最常见的职业损伤之一. 为了解在手工搬举任务中, 不同的重物 认知信息对搬举生物力学特性的影响, 招募 24 名有搬举工作经验的健康男性, 完成在不同重物认知信息 （质量提示信息和易碎性提示信息）和重物质量组合情况下的搬举任务. 重物提示信息设置了无信息、质 量信息、易碎信息、同时提示质量和易碎信息 4 个水平. 重物质量设置了轻、适中和重 3 个水平. 分析结 果显示, 在提示易碎信息的情况下, 受试人大腿和背部的肌肉活动减弱, 下背力矩及䯘关节最大角速度减 小; 随着搬举动作持续时间延长, 下背受伤的风险增高; 质量信息对下背生物力学无显著的影响; 与认知 信息相比, 重物质量对搬举生物力学表现的影响更大. 因此, 相比给予重物信息提示, 控制搬举负重是保 障搬举工人腰背健康更有效的方式. 研究结果可为制定安全手工搬举作业标准提供参考.
\end{abstract}

关键词：生物力学; 职业损伤; 下背疼痛; 搬举; 重物信息; 运动分析; 表面肌电

中图分类号：TB18；TB49 文献标志码：A doi：10.3724/SP. J. 1249.2021.03287

\section{Effects of load information on biomechanical characteristics of lifting tasks}

\author{
LIU Yipeng, LIU Junshi, and QU Xingda \\ Institute of Human Factors and Ergonomics, Shenzhen University, Shenzhen 518060, Guangdong Province, P. R. China
}

\begin{abstract}
The lower back pain caused by manual lifting is one of the most common occupational injuries. This study aims to investigate how biomechanics of lifting tasks is affected by the availability of load information. Twenty-four healthy men with lifting experience were recruited, and they were instructed to perform the lifting tasks under different weights, fragility information and lifting load conditions. The lifting load was set at three levels including light, medium and heavy. The load prompt information has four levels corresponding to "no information”, “prompt mass information", “prompt material fragility information” and "prompt mass and material fragility information”, respectively. The results show that with the availability of material fragility information, the muscle activity of thigh and back is weakened, meanwhile, the torque of low back and the maximum angular velocity of hip joint are reduced. However, due to the longer duration of lifting, the risk of low back injuries increased instead. The prompt information about load weight has no significant influence on lower back biomechanics. The lifting load effects on lifting biomechanics are more substantial than load prompt information. This suggests that reducing lifting load should be more effective in the control of lower back pain. The experimental results can provide useful guidance for the design of safe manual lifting operation standards.
\end{abstract}

Key words : biomechanics; occupational injuries; low back pain; lifting; load information; motion analysis; surface electromyography

Received: 2021-01-06; Accepted: 2021-03-23

Foundation: National Natural Science Foundation of China (31570944)

Corresponding author: Professor QU Xingda. E-mail: quxd@ szu.edu.cn

Citation: LIU Yipeng, LIU Junshi, QU Xingda. Effects of load information on biomechanical characteristics of lifting tasks [J]. Journal of Shenzhen University Science and Engineering, 2021, 38(3): 287-294. (in Chinese) 
超过 $40 \%$ 的工作者发生过与工作相关的肌肉骨 骼损伤 ${ }^{[1]}$. 其中，手工搬举作业导致的下背和腰椎 损伤大约占所有相关肌肉骨骼损伤工作的 $31 \%$. 与手工搬举作业相关的下背疼痛导致中国每年国内 生产总值损失 $1 \%{ }^{[3]}$ ，以及每 $1 \times 10^{4} \mathrm{~h}$ 工时 $23 \mathrm{~d}$ 的 因伤离岗 ${ }^{[4]}$.

手工搬举作业中，导致下背疼痛的外界物理因 素主要有过重的搬举负担、长时间重复动作、别扭 的搬举姿势 (非对称的搬举) 、不舒适的高度和搬 举频率过高等 ${ }^{[5-6]}$. 为解决搬举导致的下背疼痛问 题, 许多学者对搬举生物力学进行研究. DAVIS 等 ${ }^{[7]}$ 探讨了搬举高度对下背受力的影响, 发现重物 初始高度在膝盖高度时下背力矩最大, 在手肘高度 时下背力矩最小. PUNNETT 等 ${ }^{[8]}$ 研究了躯干角度 变大和下背疼痛的关系, 发现较大的躯干弯曲角度 会使下背受伤的概率增加. 不当的搬举姿势也会导 致下背疼痛, 与对称搬举作业相比较, 非对称搬举 作业会使腰椎旋转角和下背剪切力变大, 增加下背 疼痛的几率 ${ }^{[9]}$.

搬举工人的心理负荷被认为与外界物理负荷一 样, 都可导致肌肉骨骼的损伤 ${ }^{[9]}$. 在搬举作业中, 重物的质量和体积等外部物理特性会改变搬举者对 重物主观的负荷认知, 从而使其调整身体发力以及 动作控制策略 ${ }^{[3]}$. 从生物力学的角度来解释, 这种 由重物认知信息带来的身体调整将使下背及腰椎的 生物力学结构发生改变 ${ }^{[9]}$, 进而影响搬举作业中下 背受伤的风险. 文献 $[10]$ 研究发现, 在不知道重物 质量的情况下，搬举者会根据重物体积错误估计重 物的质量, 在搬举的初始阶段容易产生强烈的肌肉 活动, 使其肌肉发力及运动控制与实际搬举需求不 符, 进而增加下背受伤的风险. De LOOZE 等 ${ }^{[11]}$ 研 究发现, 受试人在搬举未知质量的重物时, 其躯干 力矩和躯干伸肌活动强度相比已知重物质量的情况 增加 $10 \%$. COMMISSARIS 等 ${ }^{[12]}$ 指出, 在搬举过程 中, 搬举者高估重物质量会增大下背疼痛风险, 原 因是高估重物质量会导致姿势改变，从而使下背力 矩增加. KOTOWSKI 等 ${ }^{[13]}$ 指出, 搬举者低估重物 质量时会导致身体突然前倾，为了维持身体平衡不 得不激活更大的肌肉活动和躯干力矩，导致下背的 负荷变得更大. 认知信息对搬举作业的完成时间也 有一定的影响, PATTERSON 等 ${ }^{[14]}$ 观察到受试人在 搬举提示为较重的物体时，搬举动作的持续时间会 变长, 而持续时间越长, 肌肉的负荷越大, 下背疼 痛的风险也就越大.
上述有关重物认知信息的研究仅探讨了重物的 质量信息或者体积信息对搬举生物力学的影响. 而 在日常的搬举工作中经常需要处理易碎物品, 搬举 者在移动易碎物品时通常会比较谨慎, 有可能表现 出不一样的生物力学特性, 但目前尚无关于搬举易 碎物品的生物力学研究. 因此, 本研究所检验的重 物信息种类除了质量信息外，还包括材质信息 $($ 是 否易碎). 本研究所分析的生物力学指标有下背力 矩、身体运动学、表面肌电和作业完成时间 4 项. 研究假设: (1) 在搬举作业中, 重物认知信息可导 致下背生物力学特性的改变; (2) 重物质量与重物 认知信息对下背生物力学的影响可能存在一定的交 互作用，即搬举的重物质量越大，重物认知信息造 成的生物力学改变将更明显，从而导致更高的下背 安全风险.

\section{1 研究方法}

\section{1 受试人}

实验招募了 24 名有手工搬举工作经验的健康 男性，在实验前签署知情同意书. 所有受试人的年 龄为 $20 \sim 50$ 岁, 身体质量指数 (body mass index, BMI) 为 $18 \sim 24$, 均无腰背部疾病史, 测试时无背 部疼痛, 脊椎活动度健康. 受试人的信息如表 1 , 其中, 搬举能力指受试人可接受的最大搬举质量, 计算方法见实验流程.

\section{表 1 受试者信息}

Table 1 Information of participants

\begin{tabular}{ccccc}
\hline & 年龄 $/$ 岁 & 身高 $/ \mathrm{cm}$ & 体质量 $/ \mathrm{kg}$ & 搬举能力 $/ \mathrm{kg}$ \\
\hline 均值 & 36.2 & 170.6 & 74.9 & 9.6 \\
标准差 & 8.5 & 4.6 & 9.1 & 3.4 \\
\hline
\end{tabular}

\section{2 实验设备}

由 8 个红外摄像头组成的光学运动捕捉系统 (Vicon nexus 1.3，英国 Oxford Metrics 公司）记录 受试人以及重物的运动学数据. $14 \mathrm{~mm}$ 红外反光球 39 个，根据 VICON Plug-in-Gait (https://docs. vicon. com/display/Nexus26/Full body modeling with Plug-in Gait) 全身模型贴在受试人身体的 39 处解剖 学标志点, 用于收集全身运动数据; 反光球 4 个贴 在重物的 4 个角落, 用于确定重物运动轨迹. 2 个 测力台（FP4060，美国 Bertec 公司）用于采集足底 压力数据, 然后输入逆动力学模型 ${ }^{[15]}$ 计得下背关 节的力矩. 表面肌电系统 (MR3, 美国 Noraxon 公 
司）用于收集搬举过程中肌肉的表面肌电信号, 肌 电电极放置在两侧背坚脊肌、腹直肌和大腿外股直 肌处. 运动捕捉系统、力台、肌电系统的采样同步 频率分别设置为 $100 、 1000$ 和 $1500 \mathrm{~Hz}$.

\section{3 实验流程}

在搬举任务前, 采用心理生理方法 ${ }^{[13]}$ 测试每 位受试人的最大搬举能力. 测试过程为: 先进行 2 $\min$ 热身活动; 然后完成 2 组共 $20 \mathrm{~min}$ 的模拟搬举 练习, 练习频率为 4 次 $/ \mathrm{min}$. 在练习过程中, 受试 人需要将摆放在双脚前的体积为 $30 \mathrm{~cm} \times 40 \mathrm{~cm} \times 30$ $\mathrm{cm}$ 的重物箱子, 从地面搬举并放置到 $75 \mathrm{~cm}$ 高的 架子上的指定区域; 架子和箱子相对双脚的距离 （双脚限定在力台内）由受试人在练习前自行选定, 并在整个练习过程中保持固定. 两组练习重物的初 始质量分别设为 $2 \mathrm{~kg}$ 和 $20 \mathrm{~kg}, 2$ 组练习间休息 5 min. 在练习过程中, 受试人会被问到 “按照当前 的搬举质量和频率, 是否能够在不感觉疲劳和腰酸 背痛的情况下持续工作 $8 \mathrm{~h}$ ”, 然后根据受试人的反 馈添加（初始质量为 $2 \mathrm{~kg}$ ) 或减少重物（初始质量 为 $20 \mathrm{~kg}$ ), 直到受试人的回复从 “是” 转变为 “否” (初始质量为 $2 \mathrm{~kg}$ ), 或者由 “否” 转变为 “是” (初始质量为 $20 \mathrm{~kg}$ ). 最后, 取两组转变质 量的平均值定义为受试人可接受的最大搬举质量.

为确定受试者无任何不良反应, 搬举任务在完 成心理生理测试至少一周后进行. 任务的流程是: 首先, 将受试者两侧腹直肌、坚脊肌和外股直肌的 毛发刮干净, 并贴上肌电电极; 然后, 分别对 3 个 部位肌肉进行最大自主收缩 (maximum voluntary contraction, MVC) 测试, MVC 测试在等速肌力测 试训练仪 (瑞士 CONTREX 公司) 上进行, 如图 1. 腹部与背部的测试需要受试人分别仰卧和俯卧, 仅 依靠躯干发力维持最大用力的俯卧挺身 (背部发力) 或仰卧起坐(腹部发力) 约 $5 \sim 10 \mathrm{~s}$; 腿部的测试是 让受试人采取坐姿, 绷直大腿用力发力 $5 \sim 10 \mathrm{~s}$. 收集 MVC 测试过程的表面肌电信号作为后续肌电 信号处理的标准值.

在受试人身上贴好反光球后开始搬举任务. 搬 举任务与心理生理测试练习时的搬举任务相同, 即 将重物箱子从双脚前的地面上搬到架子上的目的区 域. 箱子、架子和受试人站立的位置与心理生理测 试时一致. 本研究的自变量有重物质量和重物提示 信息. 重物质量设为轻、适中和重 3 个水平, 分别 是受试人可接受的最大搬举质量的 $40 \% 、 80 \%$ 和 $120 \%$. 重物的提示信息设为无信息 (no information,

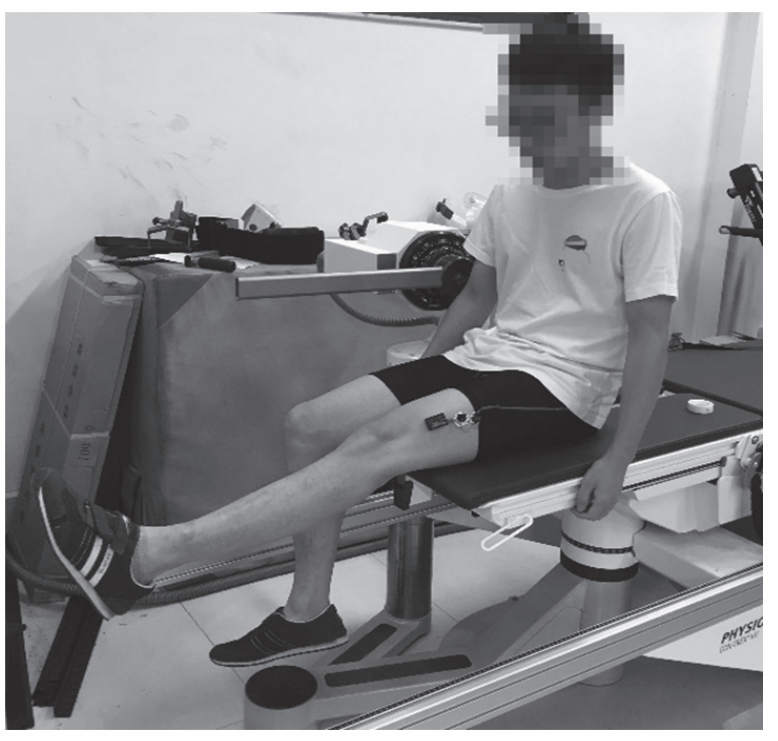

图 1 -名受试人正在进行腿部 MVC 测试 (左腿处粘贴 Noraxon 肌电仪)

Fig. 1 A participant performing thigh MVC test

(a Noraxon electromyograph pasted on left thigh)

NO) 、质量提示 ( known weight, WT)、内含易碎物 提示 (known material fragility, FRA) 以及同时提示质 量和易碎物信息 ( known weight and known material fragility, BOTH) 4 项, 如图 2. 实验人员在数据收 集前会向受试人解释重物提示信息的含义, 并有意 告知受试人箱内重物与提示信息的内容一致. 实验 共包括 12 种测试条件 (3 种质量 $\times 4$ 种提示信息). 为了抵消受试人之间相互学习效应的干扰, 实验中 首先将 3 种质量水平的实验顺序在受试人之间随机 安排, 然后在同一质量水平下, 对 4 种提示信息出 现的先后顺序也作随机安排.

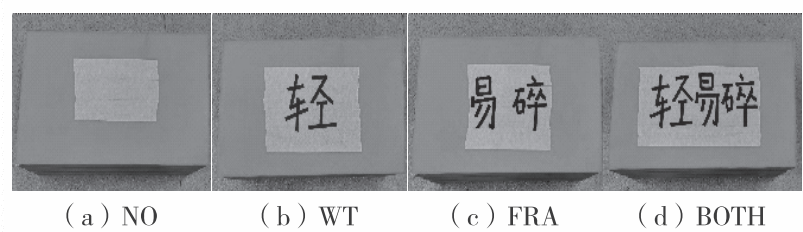

图 2 质量为轻、提示信息分别为 NO、WT、 FRA 和 BOTH 的 4 种重物

Fig. 2 Four types of load which the load weight is light and the load information is NO, WT, FRA, and BOTH

为提高收集数据的可靠性, 每种条件的任务连 续重复 3 次. 每完成一次任务休息 $2 \mathrm{~min}$, 以减小 实验中可能产生的疲劳效应. 此外, 在实验前和实 验后, 分别记录受试人的心率作为任务负荷的参 考. 图 3 为实验中受试人在完成搬举任务. 


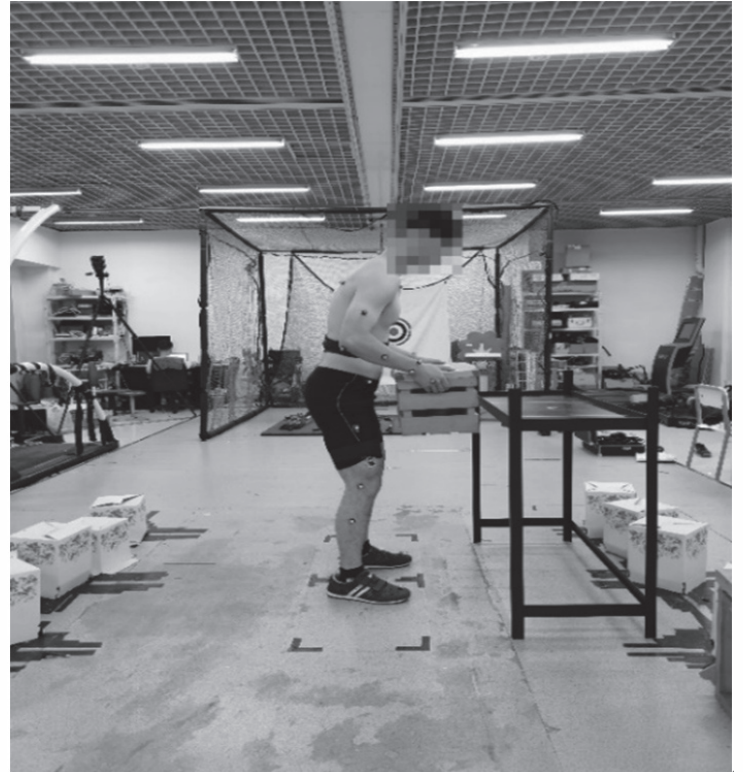

图 3 正在完成搬举任务的受试人

Fig. 3 A participant performing a lifting task in the experiment

\section{4 因变量}

搬举过程分为抬升和放置两个阶段 ${ }^{[16]}$. 抬升 阶段定义为重物开始上升到离躯干水平距离最近的 时间段. 放置阶段为重物离躯干水平距离最近到重 物到达目的位置的时间段. 抬升阶段主要依赖下肢 以及躯干发力来完成，放置阶段主要依赖上肢以及 躯干发力来完成.

实验选取 4 种类型的因变量以评估不同任务的 负荷难度: 下背力矩变量、躯干及髋关节运动学变 量、表面肌电变量和搬举动作完成的时间变量. HARARI 等 ${ }^{[17]}$ 研究指出, 下背关节力矩过大是导致 下背疼痛最直接的原因. 下背力矩指 L $5 \mathrm{~S} 1$ 关节力 矩 ${ }^{[15]}$. 下背关节的平均力矩和峰值力矩均被用作 评估搬举作业中下背疼痛的风险 ${ }^{[18]}$ ，因此，实验 选取的力矩指标包括在抬升和放置两个阶段的力矩 均值和整个搬举过程的力矩峰值.

搬举者的关节角度和关节角速度等运动学参数 描述了搬举者的姿势和动作控制策略，而躯干及髋 关节运动学表现与下背受伤有直接的关系 ${ }^{[16]}$. 因 此, 将躯干及髋关节运动学作为实验第 2 类因变 量. 实验分析的运动学指标包括结束角度 (重物被 放置到目的位置时刻的关节角度)、搬举过程的最 大角度和最大角速度. 所选取的运动学指标均属于 搬举的风险指标 ${ }^{[19]}$, 在此前的研究中也被广泛用 于分析搬举任务的负荷.

肌肉发力是造成肌肉疲劳甚至损伤的主要因
素, 表面肌电信号可以很好地反映肌肉发力状 况 ${ }^{[20]}$, 因此将表面肌电作为评估搬举任务生物力 学特性的另一因变量. 搬举作业中, 腹直肌和竖脊 肌的表面肌电信号强度可很好地反映搬举任务的用 力情况 ${ }^{[21]}$, 因此, 实验采集了两侧腹直肌、背坚 脊肌的表面肌电. 此外, 实验还收集了大腿外股直 肌的表面肌电作为腿部负荷的参考. 原始的肌电信 号都经过 Noraxon 系统集成的整流和基于海明窗的 带通滤波截止频率为 $20 \sim 300 \mathrm{~Hz}$ 处理, 系统初步 处理后的肌电信号再以 MVC 测试时的肌电峰值为 标准进行标准化处理. 在肌肉发力的过程中，瞬时 发力过大或者持续进行发力均可导致肌肉疲劳 ${ }^{[22]}$, 所以选取的肌电指标包括在抬升和放置两个阶段的 肌电均值以及搬举全过程的肌电峰值. 另外, 肌肉 发力时间越长, 肌肉受伤的风险越高 ${ }^{[9]}$, 因此, 将 完成时间也作为评估任务风险的指标.

\section{5 统计分析}

采用重复测量方差分析法对自变量 (质量和提 示信息) 及其交互效果进行分析，Bonferoni 检验法 做事后比较. 球形检验用来判断数据是否符合球形 假设，如果不符合球形假设，则使用 GreenhouseGeisser 校正后的自由度和 $P$ 值. 若质量和重物信息 存在交互作用，则在质量的各个水平下做重物信息 的事后检验. 所有分析的显著性水平 $\alpha$ 设为 0.05 . 实验中每种任务条件重复 3 次, 取 3 次实验结果的 均值作为因变量.

\section{2 结果与分析}

对实验数据分析发现，重物认知信息效应对下 背力矩、背部表面肌电、完成时间和髋关节等生物 力学参数的一些指标有显著影响, 其中提供质量信 息并未改变抬举生物力学特性, 而易碎信息的提供 对生物力学特性产生了显著影响. 另外，质量效应 对下背力矩、躯干及髋部运动学和完成时间等指标 均有显著影响. 与研究最初假设不同的是, 质量和 认知信息并无明显的交互作用.

\section{1 重物认知信息效应}

表 2 为重物认知信息效应的结果，其中，肌电 变量为标准化值, 单位为 1 . 由表 2 可见, 重物认 知信息效应对抬升阶段的下背力矩值有显著影响 $(P=0.026)$. 其中, 质量信息对下背力矩的影响较 小，易碎信息对下背力矩有显著的影响，在提示易 碎信息的任务中下背力矩相比无易碎信息时减小, 
表 2 重物认知信息效应 $(\bar{x} \pm s)^{1)}$

Table 2 Effects of weight and fragility information $(\bar{x} \pm s)$

\begin{tabular}{|c|c|c|c|c|c|c|c|c|c|c|c|c|}
\hline \multirow{2}{*}{$\begin{array}{l}\text { 重物提 } \\
\text { 示信息 }\end{array}$} & \multicolumn{5}{|c|}{ 下背力矩 } & \multicolumn{7}{|c|}{ 躯干运动学 } \\
\hline & 抬升/N·m & \multicolumn{2}{|l|}{ 放置/N·m } & \multicolumn{2}{|c|}{ 峰值/N·m } & \multicolumn{2}{|c|}{ 结束角度/( $\left.{ }^{\circ}\right)$} & \multicolumn{3}{|c|}{ 最大角度/( $\left.{ }^{\circ}\right)$} & \multicolumn{2}{|c|}{ 最大角速度 $/\left(\left({ }^{\circ}\right) \cdot \mathrm{s}^{-1}\right)$} \\
\hline NO & $164.37 \pm 34.61^{\mathrm{a}, \mathrm{b}}$ & \multicolumn{2}{|c|}{$102.16 \pm 29.03$} & \multicolumn{2}{|c|}{$208.91 \pm 39.43$} & \multicolumn{2}{|c|}{$11.22 \pm 7.75$} & \multicolumn{3}{|c|}{$38.91 \pm 12.83$} & \multicolumn{2}{|c|}{$42.09 \pm 14.88$} \\
\hline WT & $164.37 \pm 33.59^{\mathrm{a}}$ & \multicolumn{2}{|c|}{$101.91 \pm 29.28$} & \multicolumn{2}{|c|}{$208.97 \pm 38.23$} & \multicolumn{2}{|c|}{$12.01 \pm 7.70$} & \multicolumn{3}{|c|}{$38.60 \pm 12.66$} & \multicolumn{2}{|c|}{$41.38 \pm 14.15$} \\
\hline FRA & $162.57 \pm 33.10^{\mathrm{b}}$ & \multicolumn{2}{|c|}{$103.00 \pm 28.95$} & \multicolumn{2}{|c|}{$207.15 \pm 36.95$} & \multicolumn{2}{|c|}{$11.91 \pm 8.17$} & \multicolumn{3}{|c|}{$39.23 \pm 12.29$} & \multicolumn{2}{|c|}{$40.69 \pm 12.87$} \\
\hline ВОТН & $163.12 \pm 33.17^{\mathrm{a}, \mathrm{b}}$ & \multicolumn{2}{|c|}{$103.14 \pm 30.61$} & \multicolumn{2}{|c|}{$207.60 \pm 37.36$} & \multicolumn{2}{|c|}{$12.25 \pm 8.05$} & \multicolumn{3}{|c|}{$39.38 \pm 12.60$} & \multicolumn{2}{|c|}{$40.78 \pm 14.67$} \\
\hline$P$ 值 & 0.026 & \multicolumn{2}{|l|}{0.689} & \multicolumn{2}{|c|}{0.307} & \multicolumn{2}{|c|}{0.081} & & 0.397 & & & 0.642 \\
\hline 重物提 & & 髋关节运动 & & & & & & & & 背坚脊 & 肌 & \\
\hline 示信息 & 结束角度/(o) & 最大角度/( $\left.{ }^{\circ}\right)$ & 最大 & 大角速度 $/(($ & $\left.\cdot \mathrm{s}^{-1}\right)$ & & 抬升 & & & 放置 & & 峰值 \\
\hline NO & $20.79 \pm 7.10$ & $98.66 \pm 10.82$ & & $99.94 \pm 22.0$ & & 0.73 & \pm 0.24 & & 0.7 & $1 \pm 0$ & $24^{\mathrm{a}, \mathrm{b}}$ & $1.31 \pm 0.46$ \\
\hline WT & $21.24 \pm 6.96$ & $99.17 \pm 10.84$ & & $101.44 \pm 22$. & & 0.73 & \pm 0.24 & & & $72 \pm 0$. & $26^{\mathrm{a}}$ & $1.30 \pm 0.45$ \\
\hline FRA & $20.89 \pm 7.05$ & $98.01 \pm 10.75$ & & $97.60 \pm 22.8$ & & 0.72 & +0.25 & & 0.7 & $1 \pm 0$ & $27^{\mathrm{a}, \mathrm{b}}$ & $1.27 \pm 0.44$ \\
\hline ВOTH & $20.78 \pm 7.07$ & $98.53 \pm 10.93$ & & $97.06 \pm 22$. & & 0.71 & \pm 0.23 & & & $71 \pm 0$ & $26^{\mathrm{b}}$ & $1.27 \pm 0.44$ \\
\hline$P$ 值 & 0.554 & 0.060 & & 0.022 & & & 028 & & & 0.032 & & 0.101 \\
\hline 重物提 & & 腹直肌 & & & & & 大腿 & 外股 & 直肌 & & & 搬举完成 \\
\hline 示信息 & 抬升 & 放置 & & 峰值 & & 抬升 & & 放置 & & & 峰值 & 时间/s \\
\hline NO & $0.09 \pm 0.08^{\mathrm{a}}$ & $0.09 \pm 0.09$ & & $21 \pm 0.17$ & 0.52 & $2 \pm 0.17$ & 0.13 & $3 \pm 0$ & $.07^{\mathrm{a}}$ & 1.0 & $1 \pm 0.32$ & $2.11 \pm 0.26^{\mathrm{a}}$ \\
\hline WT & $0.09 \pm 0.07^{\mathrm{a}}$ & $0.09 \pm 0.09$ & & $22 \pm 0.18$ & 0.51 & $1 \pm 0.17$ & 0.13 & $3 \pm 0$ & $.07^{\mathrm{a}}$ & 1.0 & $3 \pm 0.37$ & $2.12 \pm 0.28^{\mathrm{a}, \mathrm{b}}$ \\
\hline FRA & $0.08 \pm 0.07^{\mathrm{a}}$ & $0.09 \pm 0.08$ & & $21 \pm 0.16$ & 0.50 & \pm 0.16 & 0.12 & \pm 0 & $07^{\mathrm{a}, \mathrm{b}}$ & 1.0 & $0 \pm 0.35$ & $2.19 \pm 0.29^{\mathrm{a}, \mathrm{b}}$ \\
\hline ВоTH & $0.08 \pm 0.08^{\mathrm{a}}$ & $0.09 \pm 0.08$ & & $20 \pm 0.16$ & 0.50 & \pm \pm 0.17 & 0.12 & $2 \pm 0$ & $.06^{\mathrm{b}}$ & 1.0 & $0 \pm 0.33$ & $2.20 \pm 0.28^{\mathrm{b}}$ \\
\hline$P$ 值 & 0.014 & 0.124 & & 0.481 & & .094 & & ). 04 & & & .533 & 0.005 \\
\hline
\end{tabular}

事后比较的结果显示, FRA 任务的下背力矩比 NO 任务及 WT 任务的下背力矩平均减小 $1.8 \mathrm{~N} \cdot \mathrm{m}$.

对躯干和髋关节运动学数据分析的结果表明, 重物认知信息效应仅对髋关节的最大角速度有显著 影响. 其中, 质量信息对结果的影响较小, 易碎信 息对髋关节最大角速度有显著性的影响, 事后比较 的结果表明, BOTH 任务时髋关节最大角速度相比 WT 任务时下降了 3.8\% ( $P=0.005)$, FRA 任务时 髋关节最大角度相比 NO 任务时下降了 $2.3 \%$ 。

重物认知信息效应对抬升阶段背坚脊肌的肌电 均值 $(P=0.028)$ 、抬升阶段腹直肌的肌电均值 $(P=0.032)$ 和放置阶段大腿外股直肌的肌电均值 $(P=0.044)$ 均有显著效应, 对 3 处肌肉的肌电峰 值均无显著效应. 对比不同提示信息任务的肌肉活 动情况，仅发现易碎信息对肌肉活动情况的显著影 响. 事后比较的结果表明, 在抬升和放置两个阶 段, BOTH 任务时背竖脊肌的肌电均值相比 WT 任 务时减小了约 $2.5 \%$ (抬升阶段 $P=0.019$, 放置阶
段 $P=0.029)$. 在放置阶段, 大腿肌肉活动在提示 易碎信息的任务中相比无易碎信息时显著下降，事 后分析的结果显示, BOTH 任务时大腿肌肉活动相 比 NO 任务下降 7.1\% $(P=0.023)$, 相比 WT 任务 时下降了 $8.6 \%(P=0.033)$.

重物认知信息效应对作业完成时间也有显著性 的影响 $(P=0.005)$. 其中质量信息对完成时间的 影响较小，易碎信息对完成时间有显著性的影响， 提示易碎信息的任务中的完成时间相比无易碎信息 任务变长, 事后比较的结果表明, BOTH 任务的完 成时间相比 NO 任务增加了约 4.5\% $(P=0.01)$.

\section{2 质量效应}

表 3 给出了质量效应的结果, 可以看到质量效 应对抬升和放置阶段的下背力矩值以及力矩峰值均 有显著影响 $(P<0.001)$, 下背力矩值随着搬举重 物的质量增加而显著增大. 躯干和髋关节运动学数 据分析的结果表明，质量效应对躯干结束角、髋关 节的结束角和最大角均有显著影响, 这些运动学指 
标与质量有较强的正相关性 $(P<0.005)$, 即质量 越大关节角度越大. 质量效应对背坚脊肌、腹直肌 两个阶段的肌电均值和峰值均有显著影响 $(P<$ $0.001)$ ，但仅对放置阶段的大腿肌电值有显著效应 $(P<0.001)$, 这些指标均表现出肌肉的负荷随着 质量增大而显著增大.
质量效应对搬举的完成时间有显著影响 $(P=$ $0.015)$. 质量为适中和重时的完成时间差异较小, 相比质量为轻的情况完成时间显著延长, 其中质量 为重时的完成时间相比质量轻时延长了约 $4.3 \%$ $(P=0.003)$.

表 3 质量效应 $(\bar{x} \pm s)^{1)}$

Table 3 Effects of load magnitude $(\bar{x} \pm s)$

\begin{tabular}{|c|c|c|c|c|c|c|c|c|c|c|}
\hline \multirow{2}{*}{ 重物质量 } & \multicolumn{4}{|c|}{ 下背力矩 } & \multicolumn{6}{|c|}{ 躯干运动学 } \\
\hline & 抬升/N·m & 放置 $/ \mathrm{N} \cdot \mathrm{m}$ & \multicolumn{2}{|c|}{ 峰值/N·m } & \multicolumn{2}{|c|}{ 结束角度 $/{ }^{\circ}$ ) } & \multicolumn{2}{|c|}{ 最大角度 $/\left(^{\circ}\right)$} & \multicolumn{2}{|c|}{ 最大角速度 $/\left(\left(^{\circ}\right) \cdot \mathrm{s}^{-1}\right)$} \\
\hline 轻 & $142.64 \pm 24.49^{\mathrm{a}}$ & $82.50 \pm 18.32^{\mathrm{a}}$ & \multicolumn{2}{|c|}{$185.33 \pm 29.17^{\mathrm{a}}$} & \multicolumn{2}{|c|}{$10.20 \pm 8.21^{a}$} & \multicolumn{2}{|c|}{$38.32 \pm 13.93$} & \multicolumn{2}{|c|}{$40.50 \pm 13.63$} \\
\hline 适中 & $163.51 \pm 28.32^{\mathrm{b}}$ & $102.66 \pm 23.59^{b}$ & \multicolumn{2}{|c|}{$207.84 \pm 31.90^{\mathrm{b}}$} & \multicolumn{2}{|c|}{$12.31 \pm 7.94^{\mathrm{b}}$} & \multicolumn{2}{|c|}{$39.67 \pm 12.33$} & \multicolumn{2}{|c|}{$41.85 \pm 13.64$} \\
\hline 重 & $184.67 \pm 32.95^{\mathrm{c}}$ & $122.50 \pm 29.94^{\mathrm{c}}$ & \multicolumn{2}{|c|}{$231.30 \pm 37.17^{\mathrm{c}}$} & \multicolumn{2}{|c|}{$13.0 \pm 7.28^{b}$} & \multicolumn{2}{|c|}{$39.10 \pm 11.29$} & \multicolumn{2}{|c|}{$41.37 \pm 15.08$} \\
\hline$P$ 值 & $<0.001$ & $<0.001$ & \multicolumn{2}{|c|}{$<0.001$} & \multicolumn{2}{|c|}{$<0.001$} & \multicolumn{2}{|c|}{0.292} & \multicolumn{2}{|c|}{0.632} \\
\hline \multirow{2}{*}{ 重物质量 } & \multicolumn{4}{|c|}{ 髋关节运动学 } & \multicolumn{6}{|c|}{ 背坚脊肌 } \\
\hline & 结束角度 $/\left(^{\circ}\right)$ & 最大角度 $/\left(^{\circ}\right)$ 最 & \multicolumn{2}{|c|}{ 最大角速度 $/\left(\left(^{\circ}\right) \cdot \mathrm{s}^{-1}\right)$} & \multicolumn{2}{|c|}{ 抬升 } & \multicolumn{2}{|r|}{ 放置 } & & 峰值 \\
\hline 轻 & $18.65 \pm 6.75^{\mathrm{a}}$ & $97.67 \pm 11.17^{\mathrm{a}}$ & \multicolumn{2}{|c|}{$98.42 \pm 22.74$} & 0.63 & $\pm 0.20^{\mathrm{a}}$ & & $.60 \pm 0.20$ & & $1.12 \pm 0.36^{\mathrm{a}}$ \\
\hline 适中 & $20.98 \pm 6.45^{\mathrm{b}}$ & $98.30 \pm 10.91^{\mathrm{a}}$ & $98.97 \pm 22$. & & 0.73 & $\pm 0.22^{\mathrm{b}}$ & & $.71 \pm 0.24$ & & $1.29 \pm 0.43^{b}$ \\
\hline 重 & $23.15 \pm 7.13^{\mathrm{c}}$ & $99.81 \pm 10.26^{b}$ & $99.64 \pm 21$ & & 0.81 & $\pm 0.26^{\circ}$ & & $.83 \pm 0.27$ & & $1.45 \pm 0.48^{\mathrm{c}}$ \\
\hline$P$ 值 & $<0.001$ & 0.012 & 0.824 & & & .001 & & $<0.001$ & & $<0.001$ \\
\hline & & 腹直肌 & & & & 大腿外 & 外股直肌 & & & 搬举完成 \\
\hline 里物页里 & 抬升 & 放置 & 峰值 & & 抬升 & & 放置 & 峰 & 值 & 时间/s \\
\hline 轻 & $0.07 \pm 0.06^{\mathrm{a}}$ & $0.07 \pm 0.06^{\mathrm{a}}$ & $0.18 \pm 0.14^{\mathrm{a}}$ & 0.50 & $0 \pm 0.18$ & $0.11=$ & $\pm 0.07^{\mathrm{a}}$ & $1.01=$ & \pm 0.36 & $2.10 \pm 0.26^{\mathrm{a}}$ \\
\hline 适中 & $0.09 \pm 0.07^{\mathrm{b}}$ & $0.09 \pm 0.08^{b}$ & $0.21 \pm 0.17^{\mathrm{b}}$ & 0.51 & $1 \pm 0.17$ & $0.12=$ & $\pm 0.07^{\mathrm{a}}$ & $1.01=$ & \pm 0.30 & $2.17 \pm 0.27^{\mathrm{a}, \mathrm{b}}$ \\
\hline 重 & $0.10 \pm 0.09^{\mathrm{c}}$ & $0.11 \pm 0.10^{c}$ & $0.24 \pm 0.19^{c}$ & 0.51 & $1 \pm 0.16$ & $0.14=$ & $\pm 0.07^{\mathrm{b}}$ & $1.01=$ & \pm 0.37 & $2.19 \pm 0.31^{\mathrm{b}}$ \\
\hline$P$ 值 & $<0.001$ & $<0.001$ & $<0.001$ & & 803 & & 0.001 & 0.9 & 949 & 0.015 \\
\hline
\end{tabular}

1) 字母上标 $(\mathrm{a} 、 \mathrm{~b}$ 或 $\mathrm{c})$ 相同表示无显著差异, 不同表示有显著差异; 灰底的 $P$ 值表示有显著差异 $(P<0.05)$

\section{3 讨 论}

本研究检验了不同重物认知信息与重物质量组 合情况下，搬举任务的表面肌电和下背力矩等生物 力学特性. 分析结果表明, 认知信息效应对下背生 物力学特性具有显著性的影响, 其中质量信息造成 的影响较小，易碎信息造成的影响较大. 重物质量 增加是下背生物力学负荷增加的主要原因, 因此, 相比重物认知信息效应导致的下背负荷，重物质量 增加导致的下背负荷更有可能导致下背疼痛.

\section{1 重物认知信息效应}

研究结果表明，重物认知信息效应对抬升阶段 的下背力矩表现有显著影响. 其中，质量信息对下 背力矩值的影响较小，易碎信息对下背力矩值有显
著影响. 在有易碎信息提示的任务中下背力矩值减 小, 完成 FRA 任务时的下背力矩相比完成 NO 任务 时减小了 $1.8 \mathrm{~N} \cdot \mathrm{m}$, 完成 BOTH 任务时的下背力矩 相比完成 WT 任务时减小了 $1.3 \mathrm{~N} \cdot \mathrm{m}$. 分析其原因 是，搬举者在处理易碎物品时采取了较为小心的搬 举方式，减小了下背关节发力，因此下背力矩减 小. 此外，对比质量及易碎同时提示的任务和无信 息提示任务的下背力矩情况，发现完成 BOTH 任务 时的下背力矩值相比完成 NO 任务时的下背力矩值 下降了 $1.3 \mathrm{~N} \cdot \mathrm{m}$, 该差异值与 BOTH 任务和 WT 任 务之间的差异是一致的，这个结果也说明了重物的 质量信息并不会对下背力矩有明显的影响. 与下背 力矩情况类似的是, 运动学数据分析的结果显示, 仅易碎信息造成的显著性差异，搬举者在处理易碎 物品时采取了较为小心的搬举方式, 因此动作速度 
也相应减小. 其中, FRA 任务中髋关节最大角速度 相比 NO 任务下降了 $2.3 \%$, 完成 BOTH 任务时髋 关节最大角速度相比完成 WT 任务时下降了 $4.3 \%$ 。

易碎信息对抬升和放置阶段的背部肌肉活动, 以及放置阶段的大腿肌肉活动均有显著性影响, 且 仅观察到易碎信息造成的显著影响. FRA 任务背部 肌电值比 NO 任务减小了 $1.6 \%$, BOTH 任务背部肌 电值比 WT 任务减小了 $2.5 \%$, FRA 任务大腿肌电 值比 WT 任务下降了 7.1\%, BOTH 任务大腿肌电 值比 WT 任务下降了 $8.6 \%$, 表明易碎信息使得搬 举者减少背部和腿部肌肉的发力, 其中腿部用力减 小更明显. 根据这些信息可知, 在提示易碎信息的 情况下, 肌肉负荷会变小.

重物认知信息效应对任务完成时间有显著影 响. 其中, WT 对任务完成时间影响较小, FRA 对 完成时间有显著性的影响. 无论是否提供质量信息 完成时间均为 $2.15 \mathrm{~s}$, FRA 任务完成时间比 NO 任 务增加了 $3.8 \%$, BOTH 任务完成时间比 WT 任务 增加了 4\%, BOTH 任务完成时间比 NO 任务增加了 $4.7 \%$ 。该结果表明, 搬举者的负重时间在提示易 碎信息的任务中变长了, 也就意味着关节肌肉受伤 的风险变大. 因此, 在一些需要搬运贵重物品及易 碎物品的工作场合, 搬举者下背受伤的风险可能会 增高. 相反, 在搬运非易碎的重物时, 适当的重物 信息提示, 可减少搬举者的作业完成时间, 从而降 低腰背受伤的风险.

\section{2 质量效应}

质量效应对下背力矩以及腹背部肌肉活动均有 显著影响, 随着质量增大腰背部肌肉和关节的负荷 明显增加, 这与文献 [9-10]报道的一致, 即重物质 量因素是搬举中导致肌肉和关节受伤的主要因素之 一. 实验还发现, 质量效应对抬升阶段大腿的肌电 值及肌电峰值均无显著影响, 仅对放置阶段大腿的 肌电值有显著影响, 说明了抬升阶段外部重物对身 体施加的压力大部分都被腹背部承受, 因此重物质 量对腿部肌肉活动无较大影响, 但在放置阶段, 重 物及躯干前倾使得整体的重心前移, 因此当重物质 量变大时需要更大的腿部发力来平衡重心前移.

对躯干及髋关节运动学数据的分析可发现, 躯 干的结束角度随着质量增大而显著增大. 搬举过程 中身体前倾的角度越大时, 躯干及重物质量因杜杆 作用施加在下背关节处的力就越大, 从而将导致更 高的搬举安全风险 ${ }^{[23]}$. 同时, 髋关节的结束角度 和最大角度与质量也存在很强的正相关性, 即随着
质量增大髋关节角度就越大, 髋关节角度增大同样 会使下背疼痛的风险变高 ${ }^{[16]}$.

比较不同质量情况的完成时间发现, 质量为适 中和重时的完成时间比质量为轻时显著增大, 其中 质量为重时的完成时间相比质量为轻时延长了约 4. $5 \%$, 表明重物质量变大使得搬举者的负重时间 延长了, 这与 PATTERSON 等 ${ }^{[14]}$ 的研究结果一致. 质量增加使完成时间变长的同时也使肌肉和关节的 受力变大, 这些因素均可导致下背疼痛. 分析作业 完成时间的另一研究发现是, 质量为重时的完成时 间与质量适中时的完成时间非常接近, 仅增加不到 $1 \%$, 究其原因是, 当质量超过受试人的最大可承 受质量时 ( $120 \%$ 最大可承受质量), 受试人为了减 少腰背的负荷时间, 采取快速完成任务的策略来减 小受伤的可能.

\section{3 研究成果实用意义}

研究发现, 在提示易碎信息的情况下, 搬举作 业的完成时间增加, 从而使得下背处在负重状态的 时间增加, 这将不利于下背健康. 此外质量效应对 下背力矩、背部肌肉激活程度的影响程度, 要远大 于重物认知信息效应所造成的影响. 因此, 基于本 研究的结果, 可以从两个方面帮助搬举从业者保护 职业健康: (1) 在搬举材质为非易碎、质地较结实 的重物时, 适当的重物信息提示, 可减少搬举作业 的负重时间以保护下背健康; (2) 减少搬举者的工 作负重, 是保护其下背健康的更为有效的方式.

\section{结 语}

从搬举者认知信息的角度, 探究了不同的重物 提示信息对搬举生物力学的影响. 研究结果发现, 在不同的重物认知信息下, 下背生物力学特性表现 出一定的差异, 尤其在有易碎信息提示的搬举任务 中, 搬举者会采取较为谨慎的搬举策略, 使得下背 关节和肌肉发力以及髋关节角度有不同程度的减 小. 但同时作业的完成时间却变得更长, 意味着搬 举者弯腰负荷的时间越长, 而这将使得下背受伤的 风险增高. 这一发现有效地支持了初始研究假设 (1), 但并未发现重物质量与重物认知信息之间存在 交互作用, 因此, 初始假设(2)并未得到证实.

本研究存在的局限是：重物质量效应导致的下 背负荷是搬举中最主要的负荷因素, 因此应将减少 负重作为保障搬举者职业健康的最主要方式. 此 外, 在探讨重物认知信息效应如何影响下背负荷方 
面, 并未考虑年龄因素与认知信息的交互影响. 老 年人群体的身体机能相比年轻人全面下降 ${ }^{[19]}$, 因 此, 重物认知信息效应在老龄人群中可能更明显, 未来可考虑增加老年受试人检验年龄因素的影响.

\section{基金项目：国家自然科学基金资助项目（31570944）}

作者简介: 刘一鹏 (1995-), 深圳大学硕士研究生. 研究方向: 人因工程. E-mail: a184401661@ foxmail.com

引文: 刘一鹏, 刘骏识, 曲行达. 重物认知信息对搬举任务生 物力学特性的影响 $[\mathrm{J}]$. 深圳大学学报理工版, 2021, 38 (3) : 287-294

\section{参考文献 / References :}

[ 1 ] KHALAF T M, RAMADAN M Z, RAGAB A E, et al. Psychophysiological responses to manual lifting of unknown loads [J]. PLoS One, 2021, 16(2) : e0247442.

[ 2 ] Van der HAVE A, Van ROSSOM S, JONKERS I. Squat lifting imposes higher peak joint and muscle loading compared to stoop lifting $[\mathrm{J}]$. Applied Sciences, 2019, 9(18) : 3794.

[ 3 ] DICK R B, HUDOCK S D, LU Minglan, et al. Manual materials handling $[\mathrm{M}]$. New York, USA: John Wiley \& Sons, Inc. 2016: 253-257.

[ 4 ] ALEMI M M, GEISSINGER J, SIMON A A, et al. A passive exoskeleton reduces peak and mean EMG during symmetric and asymmetric lifting $[\mathrm{J}]$. Journal of Electromyography and Kinesiology, 2019, 47(3) : 25-34.

[ 5 ] HUYSAMEN K, POWER V, OSULLIVAN L. Kinematic and kinetic functional requirements for industrial exoskeletons for lifting tasks and overhead lifting $[\mathrm{J}]$. Ergonomics, $2020,63(7)$ : 818-830.

[ 6 ] MORADI B, BARAKAT S. The association of manual load lifting tasks with the ergonomic risk factors of musculoskeletal disorders $[\mathrm{J}]$. Journal of Human Environment and Health Promotion, 2020, 6(4): 183-187.

[ 7 ] DAVIS K, MARRAS W. Load spatial pathway and spine loading: how does lift origin and destination influence low back response? [J]. Ergonomics, 2005, 48(8) : 10311046.

[ 8 ] HOY D, MARCH L, BROOKS P, et al. The global burden of low back pain: estimates from the global burden of disease 2010 study [ J ]. Annals of the Rheumatic Diseases, 2014, 73(6) : 968-974.

[ 9 ] KUMAR S. Biomechanics in ergonomics [M]. 2nd ed. [S. l. ] : CRC Press, Inc, 2007: 457-468.

[10] Van der BURG J C E, Van DIEËN J H, TOUSSAINT H M. Lifting an unexpectedly heavy object: the effects on low-back loading and balance loss $[\mathrm{J}]$. Clinical Biomechanics, 2000, 15(7) : 469-477.

[11] De LOOZE M P, BOEKEN-KRUGER M C, STEENHUIZEN S, et al. Trunk muscle activation and low back loading in lifting in the absence of load knowledge $[\mathrm{J}]$. Ergonomics, 2000, 43(3) : 333-344.

[12] COMMISSARIS D M, TOUSSAINT H M. Load knowledge affects low-back loading and control of balance in lifting tasks [J]. Ergonomics, 1997, 40(5): 559-575.

[13 ] KOTOWSKI S E, DAVIS K G, SHOCKLEY K. Impact of order and load knowledge on trunk kinematics during repeated lifting tasks $[\mathrm{J}]$. Human Factors, 2007, 49 (5) : 808-819.

[14] PATTERSON P, CONGLETON J, KOPPA R, et al. The effects of load knowledge on stresses at the lower back during lifting $[\mathrm{J}]$. Ergonomics, 1987, 30 (3) : 539549.

[15] KOOPMAN A S, KINGMA I, FABER G S, et al. Estimating the L5S1 flexion/extension moment in symmetrical lifting using a simplified ambulatory measurement system [J]. Journal of Biomechanics, 2018, 70(3) : 242-248.

[16] PLAMONDON A, LARIVIÈRE C, DENIS D, et al. Difference between male and female workers lifting the same relative load when palletizing boxes $[\mathrm{J}]$. Applied Ergonomics, 2017, 60: 93-102.

[17] HARARI Y, RIEMER R, BECHAR A. Differences in spinal moments, kinematics and pace during single-task and combined manual material handling jobs $[\mathrm{J}]$. Applied Ergonomics, 2019, 81(3): 102871.

[18 ] COENEN P, KINGMA I, BOOT C R, et al. Cumulative low back load at work as a risk factor of low back pain: a prospective cohort study $[\mathrm{J}]$. Journal of Occupational Rehabilitation, 2013, 23(1): 11-18.

[19] SONG Jiahong, QU Xingda. Effects of age and its interaction with task parameters on lifting biomechanics $[\mathrm{J}]$. Ergonomics, 2014, 57(5): 653-668.

[20] TOTAH D, OJEDA L, JOHNSON D D, et al. Low-back electromyography ( EMG) data-driven load classification for dynamic lifting tasks $[\mathrm{J}]$. PLoS One, 2018, 13 (2) : e0192938.

[21] RANAVOLO A, VARRECCHIA T, IAVICOLI S, et al. Surface electromyography for risk assessment in work activities designed using the "revised NIOSH lifting equation” $[\mathrm{J}]$. International Journal of Industrial Ergonomics, 2018, 68(3) : 34-45.

[22] KENDALL F P, MCCREARY E K, PROVANCE P G, et al. Muscles: testing and function, with posture and pain [ M ]. Philabelphia, USA: Lippincott Williams \& Wilkins, 2013: 133-142.

[23] STAVE G M, WALD P H. Physical and biological hazards of the workplace $[\mathrm{M}]$. New York, USA: John Wiley \& Sons, Inc, 2016: 87-92.

【中文责编：晨 兮; 英文责编：木 柯】 